\title{
¿HACIA DÓNDE VAN LAS POLítICAS PÚBLICAS DE EDUCACIÓN SUPERIOR EN VENEZUELA?
}

\author{
HOW FAR WILL THE PUBLIC POLICIES OF \\ HIGHER EDUCATION IN VENEZUELA?
}
ATÉ ONDE VÃO AS POLÍTICAS PÚBLICAS DE EDUCAÇÃO SUPERIOR NA VENEZUELA?

\section{María Cristina Parra-Sandoval ${ }^{1}$}

\begin{abstract}
RESUMEN
En el marco del papel clave de la educación superior para el desarrollo de la sociedad, entendemos la política pública en este campo, como el conjunto de toma de decisiones y no-decisiones, con relación al sector terciario de la educación del país, por parte del gobierno o de las instituciones. Así mismo, asumimos que el proceso de establecer una política pública - en cualquier campo- supone tanto su diseño e implementación como la evaluación de sus resultados. Partiendo de estas premisas, en este capítulo se expone la contextualización histórica y los antecedentes de las políticas de educación superior en Venezuela, en los últimos doce años (19992011), para poner de relieve la particular característica que ella asume en este país durante ese periodo. Solo teniendo el panorama que ofrece este contexto y sus antecedentes, como el escenario para el diseño e implementación de las políticas de educación superior, es posible entender o aproximarse a la descripción de los factores que las determinan y sus características. En el capítulo -en la medida de lo posible, dada la carencia de información confiable- nos aproximamos a la evaluación de los resultados de las políticas implementadas y sus posibles implicaciones futuras. En Venezuela los hechos indican que la tendencia del núcleo conceptual de las políticas públicas se orienta hacia más control e intervención del Estado y apuesta al establecimiento de condiciones que tienden a la paralización del mercado universitario; mientras que, en la mayor parte de la región e incluso del mundo, la tendencia que se observa está marcada por el discurso de la mercadización y su impacto en el rol del Estado, en su tratamiento de la educación superior.
\end{abstract}

PALABRAS-CLAVE: Políticas públicas. Educación superior. Papel del estado. Venezuela

\begin{abstract}
In the framework of the key role of higher education for the development of society, we understand the public policy in this field as the decision-making assembly and non-decisions with respect to the tertiary sector of the country's education, by the government or institutions. Also, we assume that the process of establishing a public policy - in any field - assume both its design and implementation and evaluation of its results. Starting from these premises, this chapter explains the historical context and background of higher education policies in Venezuela in the last twelve years (1999-2011), to highlight the particular feature that it takes this country during that period. Only with the panorama that offers this context and its history, as the setting for the design and implementation of higher education policies, it is possible to understand or approach to the description of the factors that determine them and their characteristics. In this work - as far as possible, given the lack of reliable information - we approach the evaluation of the results of the policies implemented and their possible future implications. In Venezuela, the facts indicate that the trend of the core concept of public policy is oriented towards more control and state intervention and focus on the establishment of conditions that tend to strike the college market; whereas in most of the region and even the world, the trend observed is marked by the discourse of commodification and its impact on the state's role in their treatment of higher education.
\end{abstract}

\footnotetext{
${ }^{1}$ Socióloga. Doctora en Estudios del Desarrollo. Centro de Estudios del Desarrollo/CENDES, Universidad Central de Venezuela. Caracas, Venezuela. Profesora Titular de la Universidad del Zulia. Maracaibo, Venezuela. E-mail: mariacristina.parrasandoval@gmail.com - ORCID: http://orcid.org/0000-0001-9709-5197 Submetido em: 27/02/2016 - Aceito em: 14/04/2016

\begin{tabular}{l|l|l|l|l|l|l} 
(C) Rev. Inter. Educ. Sup. & Campinas, SP & v.2 & n.1 & p.67-88 & jan./abr. 2016 & ISSN 2446-9424
\end{tabular}
}


KEYWORDS: Public policies. Higher education. Role of the state. Venezuela

\section{RESUMO}

No marco do papel chave da educação superior para o desenvolvimento da sociedade, entendemos a política pública neste campo como o conjunto de tomadas de decisão e não-decisões, com relação ao setor terciário da educação do país, por parte do governo ou das instituições. Igualmente, assumimos que o processo de estabelecer uma política pública - em qualquer campo - supõe tanto seu desenho e implementação como a avaliação de seus resultados. Partindo destas premissas, neste capítulo se expõe a contextualização histórica e os antecedentes das políticas de educação superior na Venezuela nos últimos doze anos (1999-2011), para destacar a particular característica que ela assume neste país durante esse período. Somente tendo o panorama que oferece este contexto e seus antecedentes, como o cenário para o desenho e implementação das políticas de educação superior, é possível entender ou aproximar-se à descrição dos fatores que as determinam e suas características. Neste trabalho - na medida do possível, dada a carência de informação confiável - nos aproximamos da avaliação dos resultados das políticas implementadas e suas possíveis implicações futuras. Na Venezuela, os fatos indicam que a tendência do núcleo conceitual das políticas públicas se orienta para mais controle e intervenção do Estado e aposta no estabelecimento de condições que tendem à paralisação do mercado universitário; enquanto que, na maior parte da região e inclusive do mundo, a tendência que se observa está marcada pelo discurso da mercadorização e seu impacto no papel do Estado, em seu tratamento da educação superior.

PALAVRAS-CHAVE: Políticas públicas. Educação superior. Papel do estado. Venezuela

\section{Introducción}

Durante los últimos doce años, Venezuela ha experimentado un conjunto de cambios en todas las esferas de la vida del país. Estos cambios han sido promovidos e implementados por iniciativa del poder ejecutivo, bajo el liderazgo del Presidente Hugo Chávez. Desde la promulgación de una nueva Constitución Nacional, hasta las últimas medidas legislativas tomadas por la Asamblea Nacional, controlada mayoritariamente por miembros del partido de gobierno, la institucionalidad del país ha sido objeto de modificaciones importantes.

En el ámbito de la educación superior y, a partir de haber establecido en la Constitución Nacional $(1999)^{2}$ el principio según el cual la educación en general y, la universitaria en particular, es un derecho humano y un deber social fundamental, así como un servicio público, de cuya garantía debe rendir cuentas el Estado, las políticas implementadas han sido orientadas hacia la concreción del citado principio constitucional, teniendo como premisa el derecho de todos los habitantes “...a una educación integral, de calidad, permanente, en igualdad de condiciones y oportunidades, sin más limitaciones que las derivadas de sus aptitudes, vocación y aspiraciones." (República Bolivariana de Venezuela, 1999), para lo cual las instituciones del Estado deben ofrecerla de manera gratuita hasta el pregrado universitario.

\footnotetext{
${ }^{2}$ Esta Constitución derogó la que había sido aprobada en 1961, posterior a la caída de la dictadura de Marcos Pérez Jiménez

\begin{tabular}{l|l|l|l|l|l|l} 
(C) Rev. Inter. Educ. Sup. & Campinas, SP & v.2 & n.1 & p.67-88 & jan./abr. 2016 & ISSN 2446-9424
\end{tabular}
}


Como "norma suprema y fundamento del ordenamiento jurídico del país", la Constitución sintetiza la concepción filosófica, axiológica, económica y socio-política ${ }^{3}$ que ha permeado el desarrollo de varias etapas, en el sector de la educación superior, bastante bien demarcadas, a lo largo de todo el proceso de cambios, promovidos desde el inicio del gobierno ${ }^{4}$. La constante en cada una de ellas ha sido la profundización del proceso de refundación y reinstitucionalización de la república, para lo cual el acceso irrestricto y la gratuidad se han declarado como condiciones insoslayables en la formulación de las políticas de educación superior, durante el periodo 1999-2011.

A partir de estas premisas, en este trabajo se analizarán las políticas públicas más importantes en el campo de la educación superior, tomadas durante las tres etapas en las cuales puede dividirse ese periodo (1999-2011), teniendo en cuenta que un análisis más exhaustivo de las distintas fases de la toma de decisiones o de no-decisiones como políticas públicas, en Venezuela, se enfrenta al obstáculo de la falta de información completa y verificable en los órganos del gobierno central ${ }^{5}$ encargados de su diseño e implementación.

En ese sentido, teniendo como telón de fondo la declaración de principios contenida, tanto en la Constitución Nacional como en la Ley Orgánica de Educación ${ }^{6}$, y algunos documentos oficiales, nos aproximaremos al análisis de los factores que han incidido en las políticas durante el periodo, y en cada una de las tres etapas.

\section{Una breve reseña acerca de los antecedentes}

En 1958 termina en Venezuela la última dictadura del siglo $\mathrm{XX}$, dando inicio a un nuevo periodo durante el cual se buscó consolidar la democracia representativa, como el

\footnotetext{
${ }^{3}$ No obstante, a partir de 2007 como se expone más adelante en este trabajo, los preceptos constitucionales han sido matizados por la introducción de los postulados de lo que se ha llamado 'socialismo del siglo XXI'.

${ }^{4}$ Hay que aclarar que el Presidente Chávez fue electo por primera vez en 1998, ratificado en 2000 para "relegitimar" su elección bajo la nueva Constitución y, nuevamente reelecto en 2006, cuando inicia su segundo periodo de gobierno.

${ }^{5}$ Llama la atención que en Abril de 2011 la página web del Ministerio del Poder Popular para la Educación Universitaria informara lo siguiente con relación a las estadísticas del sector: "Con relación a los boletines estadísticos de educación universitaria, se informa que debido a una revisión de las cifras de las variables: nuevos inscritos, egresados, personal académico, personal administrativo y obrero, la publicación de éstos en la página web de la OPSU se suspenderá hasta nuevo aviso. Se recomienda visitar la página web del Ministerio del Poder Popular para la Educación Universitaria en la dirección http://www.mppeu.gob.ve http://www.mppeu.gob.ve/ para consultar la variable matrícula"; en julio del mismo año, la leyenda en el vínculo de la página dice lo siguiente: “...actualmente está disponible información relativa a la matrícula estudiantil del sector universitario a nivel de institución, correspondiente al período 1960-2008. Las cifras relativas al año 2008 son preliminares, ya que aún se está en proceso de recolección. Progresivamente, se irán incorporando otras variables, como Nuevos Inscritos, Egresados, Presupuesto y Personal Académico, Administrativo y Obrero." (http://www.mppeu.gob.ve/estadisticas.php\#). Por otra parte, toda solicitud de información estadística tiene que ser aprobada por la Dirección de Estadística y Análisis Prospectivo del MPPEU. Asimismo hay que advertir que la página sufre constantes modificaciones.

${ }^{6}$ La vigente Ley Orgánica de Educación aprobada en 2009, establece de manera explícita asuntos vinculados con la educación universitaria que no habían sido contemplados en la derogada Ley Orgánica de Educación (1980), como se detalla más adelante.
}

\begin{tabular}{l|l|l|l|l|l|l} 
(C) Rev. Inter. Educ. Sup. & Campinas, SP & v.2 & n.1 & p.67-88 & jan./abr. 2016 & ISSN 2446-9424
\end{tabular}


sistema que permitiría la democratización y modernización del país. Proceso viable gracias a la renta petrolera que le permitió al Estado constituirse en mediador social y garante del igualitarismo, para lo cual también se contaba con el consenso político necesario. Esto, en términos de la educación superior se tradujo en la garantía de acceso irrestricto, la gratuidad y, en consecuencia, en la instauración de la educación - en particular, la universitaria - como mecanismo de ascenso social y de formación de las capas medias profesionales del país (Parra Sandoval, 2010a). Sin embargo, tanto el consenso político como la renta petrolera, bases sobre las cuales se afincaba el proyecto modernizador y democrático, se deterioraron, iniciándose así un proceso de descalabro social y económico ${ }^{7}$, que culminó en 1998 con la elección del candidato cuyo programa de gobierno ofrecía a la población una salida política alternativa, ante la incapacidad de quienes habían ejercido el poder hasta entonces.

\section{El contexto de formulación de las políticas en el periodo 1999-2011}

Desde la inauguración del periodo presidencial que se inicia en 1999, se plantea la reestructuración institucional y la refundación de la república en el marco de la democracia participativa, como antagónica de la democracia representativa, característica del periodo anterior (1958-1998).

Este proceso ha estado caracterizado por una polarización política que se ha acentuado cada vez más, dividiendo a la población en dos grandes sectores irreconciliables, con escasos puntos de encuentro y/o coincidencias en cuanto al modelo de país deseado. Tal polarización ha llevado al gobierno y a los sectores que lo apoyan, primero, a profundizar y radicalizar la reestructuración institucional y la refundación de la república para el cumplimiento de las tareas y obligaciones de la administración pública y segundo, a desconocer a instituciones o personas que asuman una posición contraria a las directrices emanadas desde los organismos públicos, en función de la mencionada reestructuración y refundación y puestos al servicio de sus estrategias políticas de afianzamiento en el poder, con las consiguientes consecuencias que ello acarrea; por otra parte, se encuentran los sectores identificados con la oposición, en general menos radicalizados hoy en día, pero entre quienes aún persisten “...actores apostando al juego polarizador: algunos medios privados de comunicación, algunas organizaciones sociales y los partidos políticos, sobre todo en las coyunturas electorales." (López Maya, 2010: 103). Finalmente, a partir de 2007, sin que hubiese mediado un desarrollo previo de las fuerzas sociales, generador del consenso necesario para un cambio sociopolítico de tal envergadura, se decreta la construcción de la sociedad socialista ${ }^{8}$, con lo

\footnotetext{
${ }^{7}$ Algunos de los rasgos que caracterizaron tal descalabro, incrementados especialmente en la última década del siglo XX, fueron: creciente endeudamiento interno y externo, devaluación de la moneda, fuga de capitales, revueltas populares, golpes de Estado, aumento de la pobreza (De Venanzi, 2010).

${ }^{8}$ En diciembre de 2007 se somete a referéndum la modificación de la Constitución Nacional aprobada en 1999. Entre los cambios más importantes propuestos estaba el declarar la adhesión de Venezuela al socialismo como sistema político, sin embargo los resultados de la consulta fueron contrarios a las reformas propuestas y, en consecuencia, tal declaración fue rechazada por la mayoría de la población. No obstante, el gobierno haciendo caso omiso de ello, declara que Venezuela es socialista.
} 
cual se agudiza la polarización, en la medida en que el gobierno la utiliza como un instrumento de imposición del modelo socialista que han llamado del siglo XXI, así como para el fortalecimiento en el poder del liderazgo del Presidente Chávez (López Maya, 2010).

En este lapso de doce años (1999-2011) como ya ha sido mencionado, se puede observar el paso de varias etapas que, en el caso de la educación superior, están caracterizadas por alineaciones diferentes en cuanto al acento que la dirigencia política oficial le ha dado al contenido ideológico del proceso de reinstitucionalización, aunque mantienen principios comunes, tales como la equidad, la pertinencia y la educación superior como bien público, los cuales han incidido en la formulación de distintas políticas en el campo de la educación universitaria.

\section{Primera etapa: 1999-2002}

Esta primera etapa estuvo marcada por la euforia del triunfo obtenido en las elecciones de 1998, reafirmado en 2000, con gran apoyo popular y de las capas medias, lo cual garantizaba el respaldo a las medidas que deberían responder a las expectativas de cambio generadas durante la campaña electoral. En el campo de la educación superior, las primeras propuestas hechas llamaban a “...organizar de forma definitiva el sistema de educación superior y a impulsarlo hacia las transformaciones necesarias que la sociedad y el sector reclaman, desde hace mucho tiempo, para mejorar su calidad y equidad." (Navarro Díaz, 2001).

En tal sentido, las políticas tuvieron como meta recuperar el espacio perdido por el Estado como órgano rector de la educación superior, por su sometimiento a un paradigma neo-liberal que, de acuerdo con los análisis “...previos realizados por muchos(as) investigadores(as) de nuestras universidades, donde se expresaron los problemas de la educación superior de la región y, en particular, de Venezuela..." (Castellano, 2010: 386), había favorecido la privatización del sector, “...el afianzamiento de la concepción científicopositivista, la exasperación de la razón técnica como tecnicismos...” (Castellano, 2010:379).

En conexión con ello, una de las primeras medidas adoptadas fue la creación en 2000 del Vice-Ministerio de Educación Superior, como dependencia del Ministerio de Educación, Cultura y Deportes; desde el nuevo organismo se formularon las políticas y estrategias para el desarrollo de la educación superior, a ser llevadas a cabo en el lapso 2000-2006. Esta medida reconocía la importancia vital de este nivel educativo para la prosecución del proyecto de país que la Constitución encarna, al elevar de categoría el tratamiento del tema de la educación superior; en este sentido, puede advertirse que hubo un desarrollo del órgano de gobierno encargado de regular lo relacionado con el sector, cierta diferenciación de los organismos gubernamentales facultados para diseñar e implementar las políticas, así como la especialización de agentes y de saberes específicos (Cox, 1993). Esto último se materializó

\begin{tabular}{l|l|l|l|l|l|l} 
(C) Rev. Inter. Educ. Sup. & Campinas, SP & v.2 & n.1 & p.67-88 & jan./abr. 2016 & ISSN 2446-9424
\end{tabular}


en la presencia en funciones de gobierno, de destacados académicos universitarios, quienes no solo habían ocupado importantes cargos en la Universidad Central de Venezuela, sino que poseían reconocida experiencia de investigación en el campo de la educación superior, se habían destacado por sus críticas a las actuaciones de los gobiernos anteriores y quienes además, adherían los principios del gobierno instaurado en 1999 (García Guadilla, 2005).

Los hilos conductores de las políticas diseñadas durante esta etapa, por una parte, expresan la concepción de la educación superior como servicio público y bien social común, subyacente en la Constitución Nacional. Por otra parte, se concentran en el reconocimiento de la necesidad de incrementar la presencia del Estado, como forma de garantizar el acceso de todos al conocimiento, construir la democracia participativa y hacer que la educación sea de mayor calidad y pertinencia social; así mismo, los criterios orientadores de las políticas de educación superior de esta etapa remiten a la necesidad de garantizar el ejercicio del pensamiento crítico, la igualdad de condiciones y oportunidades educativas, la pertinencia social, la formación integral, el fortalecimiento de lo académico, en un clima de autonomía, articulación vertical y horizontal de las instituciones y de cooperación internacional (Castellano, 2001).

Sobre esta base, el Vice-Ministerio de Educación Superior formuló las siguientes políticas (Ministerio de Educación, Cultura y Deporte, 2001):

$\checkmark \quad$ Estructurar el sistema de educación superior y sus instituciones, propósitos y niveles, para la transferencia de los estudiantes y los profesores entre instituciones y niveles.

$\checkmark \quad$ Elevar la calidad académica de las instituciones y mejorar su eficiencia institucional.

$\checkmark \quad$ Mejorar la equidad en el acceso y en el desempeño de los estudiantes.

$\checkmark \quad$ Lograr una mayor pertinencia social de la educación superior en los distintos ámbitos territoriales.

$\checkmark \quad$ Lograr una mayor interrelación de las instituciones con las comunidades del entorno.

$\checkmark \quad$ Promover y fortalecer la cooperación nacional e internacional entre las instituciones de educación superior.

Para llevar adelante tales políticas, se diseñaron un conjunto de estrategias, entre las cuales es importante destacar:

a. La creación de las redes regionales de educación superior, cuyo objetivo era vincular las instituciones entre sí y con los gobiernos locales y regionales, de manera que las actividades académicas no estuviesen desvinculadas de las necesidades de las comunidades, al tiempo que se hacía viable la transferencia interinstitucional de estudiantes y profesores y se promovía una cultura de la cooperación. 
b. El Proyecto "Alma Mater" para el Mejoramiento de la Calidad y la Equidad de la Educación Universitaria, dirigido a elevar el nivel de calidad de las instituciones y al logro de la excelencia, mediante el establecimiento de: un sistema de evaluación y acreditación institucional, un sistema de asignación, seguimiento y control presupuestario, un sistema de carrera académica y, del fortalecimiento institucional. Con tal finalidad, el Proyecto preveía otorgar becas a profesores universitarios para la realización de estudios de doctorado y becas a estudiantes de escasos recursos para llevar a cabo estudios de pregrado, fortalecer las unidades de investigación y dotar de bibliotecas y salas de computación a las instituciones (Castellano, 2010). Por otra parte, el Proyecto contemplaba la creación de Aldeas Universitarias Bolivarianas cuyo objetivo era desarrollar diferentes programas académicos, administrados por distintas instituciones en un mismo espacio geográfico, ubicado en los sectores urbanos más deprimidos o en zonas rurales alejadas de los centros urbanos (Fuenmayor Toro, 2004). Finalmente, con relación al siempre álgido tema presupuestario, la estrategia que el Proyecto Alma Mater propuso consistió en el diseño de “...un modelo de presupuesto calculado sobre bases técnicas..." que debería sustituir al criticado modelo de asignaciones incrementales (García Guadilla, 2006: 9).

c. El diseño e implementación de un programa nacional de orientación profesional dirigido a lograr la equidad en el acceso a la educación superior y la optimización del desempeño de los estudiantes universitarios, mediante la revisión de las carreras ofrecidas y el análisis de su pertinencia frente a las nuevas exigencias del desarrollo nacional. Asimismo, el proyecto planteaba modificar el sistema nacional de admisión en la educación superior, establecer un sistema de auxilio financiero y atender los factores que afectan el desempeño (República Bolivariana de Venezuela. Ministerio de Educación Superior. Consejo Nacional de Universidades. Oficina de Planificación del Sector Universitario, 2002).

d. Por último, la creación de redes académicas con visión de integración latinoamericana con el objetivo de alcanzar la cooperación internacional (Consejo de Redacción, 2004).

Es indudable que tales políticas y estrategias estaban formuladas para atender las necesidades más apremiantes de la educación superior y plasmaban las propuestas surgidas de años de discusión y análisis, por parte de quienes tuvieron en esa etapa la tarea de diseñarlas; sin embargo, el análisis de sus resultados, permite señalar que los logros fueron muy parciales.

A pesar del apoyo popular con el cual contaba el gobierno, existía un clima de mutua desconfianza entre este y las universidades, especialmente las autónomas (García Guadilla, 2005). Estas universidades, que además son las más antiguas del país, eran continuamente cuestionadas por el gobierno nacional por no asumir las transformaciones internas necesarias, de acuerdo con los retos de las políticas diseñadas desde el Vice Ministerio de Educación Superior, por lo que no estarían en condiciones de responder a los requerimientos de estas políticas (González, 2008), en parte porque, tal como lo expresó quien se desempeñaba en ese momento como Vice-Ministra de Educación Superior “... las universidades fueron muy

n. 1

p.67-88

jan./abr. 2016

ISSN 2446-9424 
lentas y muy resistentes al cambio. Lo aceptaban teóricamente pero a la hora de actuar no actuaban con la rapidez que exige el proceso revolucionario." (Consejo de Redacción, 2004:52).

De tal manera que, aunque el diseño de las políticas, estrategias y acciones propuestas desde el gobierno nacional en esta etapa (1999-2002), pretendía dar respuesta a los problemas más importantes de la educación superior en Venezuela, tales políticas no lograron implantarse de manera definitiva ni producir las reformas deseadas (Parra Sandoval, 2010b).

Aparte de lograr un ligero incremento de solo $4.5 \%$ de la matrícula de las instituciones públicas entre 1999 y 2002 y del aumento en el número de instituciones del sector oficial, con la creación de tres institutos universitarios y tres universidades ${ }^{9}$ en el periodo, no llegó a constituirse el sistema de educación superior ya que, al contrario, se inició en esa etapa el proceso de diferenciación de las instituciones, basado no en condiciones institucionales o académicas, sino en los antagonismos políticos producto del proceso de polarización que también comenzaba a asomarse con nitidez en el país ${ }^{10}$. Ello impidió que pudiera darse la integración de las redes regionales de educación superior, en la medida en que no solo las universidades autónomas y algunas experimentales ${ }^{11}$ que no eran controladas por el gobierno, sino las autoridades locales en varios estados del país son contrarias al poder ejecutivo nacional y, en tanto tal dada la polarización, considerados como enemigos mas no adversarios políticos, lo cual se constituye en un fuerte obstáculo para lograr la integración deseada, al vincular la implementación de la estrategia con la confrontación política presente en la sociedad.

Por otra parte, no fue posible tampoco desarrollar las estrategias que perseguían la elevación de la calidad ni la pertinencia de la formación ofrecida en las distintas carreras. El sistema de evaluación, a cuyo diseño se había dedicado grandes esfuerzos, por parte de todas las instituciones de educación superior que lograron vincularse para elaborar la propuesta, así como el proyecto de carrera académica, fueron suspendidos. La concepción de aldeas

\footnotetext{
${ }^{9}$ Este dato puede ser verificado en la página web del Ministerio del Poder Popular para la Educación Universitaria, en el Registro Nacional de Instituciones, Programas, Grados y Titulaciones de Educación Universitaria (http://www.mppeu.gob.ve/rnipgt/autorizada.php). Al respecto es necesario señalar que dos de las Universidades que se registran como creadas en ese periodo, en realidad ya existían; una, como Universidad privada, hasta que la asumió el gobierno, otra, como Instituto Tecnológico transformado en universidad.

10 Diferenciación que posteriormente se profundiza hasta llegar a constituir dos bloques perfectamente identificados incluso con sus propias organizaciones de agrupación de los rectores de las universidades y de los sindicatos y asociaciones de los distintos miembros de las instituciones. Así hay una Asociación Venezolana de Rectores Universitarios (AVERU) que agrupa a las instituciones autónomas, algunas experimentales y privadas cuyo denominador común es que no son controladas por el gobierno y la Asociación de Rectores Bolivarianos (ARBOL) que agrupa a los Rectores de las universidades en las cuales el gobierno nombra a las autoridades.

${ }^{11}$ Las universidades experimentales, a diferencia de las autónomas, no gozan de autonomía desde su creación y actualmente solo algunas de ellas - las más antiguas- poseen esta condición. La mayoría son controladas directamente por el Ministerio del Poder Popular para la Educación Superior, quien nombra como autoridades de esas instituciones a profesores identificados políticamente con el gobierno. Aunque hay que reconocer que esta modalidad ha sido una constante desde que en 1958 fue creada la primera universidad experimental (Parra Sandoval, 2010a).
}

\begin{tabular}{l|l|l|l|l|l|l} 
(C) Rev. Inter. Educ. Sup. & Campinas, SP & v.2 & n.1 & p.67-88 & jan./abr. 2016 & ISSN 2446-9424
\end{tabular}


universitarias fue modificada y de ser un proyecto integrador de instituciones, pasó a ser el proyecto bandera de una iniciativa, la Misión Sucre, una de las políticas que comenzaron a formularse en 2003. Y, finalmente aunque no menos importante, el problema de las insuficiencias del presupuesto universitario y la forma ineficiente como se estipulan los recursos para las instituciones a través de asignaciones incrementales sin asidero técnico, continuó sin ser resuelto.

\section{Segunda etapa: 2003-2006}

El año 2002 fue un año de profunda crisis y confrontación política en el país. Intentos de golpe de Estado, manifestaciones violentas, paro petrolero, marcaron una inflexión en la vida institucional de Venezuela.

Ese año en el campo de la educación universitaria es importante destacar la creación del Ministerio de Educación Superior, al cual se atribuye la formulación, regulación y seguimiento de políticas, la orientación, programación, desarrollo, promoción, coordinación, supervisión y el control y evaluación del nivel de educación superior (República Bolivariana de Venezuela. Presidencia de la República, 2002). La creación de este Ministerio, prueba de la jerarquía más alta que se le estaba dando a la educación superior en la administración pública, es el preámbulo de las políticas que, a partir de 2003, comienzan a diseñarse, teniendo como objetivo, declarado como prioritario, garantizar la universalización de la educación superior. No obstante, a diferencia de la etapa anterior, los funcionarios encargados de la conducción de estas políticas en el periodo 2003-2006, no tenían ni la experiencia ni la solidez académica, que sí poseían quienes le precedieron, en tanto en las credenciales tomadas en cuenta para su nombramiento, prevaleció la lealtad al proceso revolucionario y al Presidente de la República.

Así, en el marco de la reacción del gobierno nacional al clima de agudización de la polarización ya instalada en el país, se implementaron un conjunto de políticas sociales dirigidas a los sectores populares, a través de los programas que llamó Misiones ${ }^{12}$; de ellas interesa destacar la Misión Sucre por el papel que esta ha tenido en el incremento del número

\footnotetext{
${ }^{12}$ Las Misiones son programas diseñados con el objeto de dar respuesta a necesidades prioritarias de educación, salud, vivienda, empleo y alimentación que todavía en ese momento no eran satisfechas por las estructuras formalmente establecidas en la administración pública central, por lo cual pueden considerarse como la concreción de la nueva institucionalidad, en tanto añaden operatividad al funcionamiento del gobierno ya que funcionan con recursos asignados directamente sin que su manejo esté sujeto a la pesada cadena de controles de la administración pública, su personal responde directamente al poder ejecutivo central y facilitan la expansión ideológica y la legitimación del proyecto del gobierno (Parra-Sandoval, Bozo de Carmona e Inciarte González, 2010). Por otra parte, también han sido señaladas como"... mecanismo de mercadeo político, útil para apuntalar la legitimación alcanzada por el presidente Chávez en el poder político, como las diferentes decisiones dirigidas a disminuir y controlar el gasto público en dichos programas, se levantan como hechos y situaciones sustanciales para enfocar el análisis de estos como una estrategia de clientelismo político y, por tanto, establecer su rendimiento a partir de la captación de votos." (Mundó, 2009: 4).
}

\begin{tabular}{|l|l|l|l|l|l|l} 
(C) Rev. Inter. Educ. Sup. & Campinas, SP & v.2 & n.1 & p.67-88 & jan./abr. 2016 & ISSN 2446-9424
\end{tabular}


de estudiantes de educación superior (Castellano, 2011) al saldar lo que se consideraba era la deuda social contraída con los que habían sido excluidos de la enseñanza universitaria: aproximadamente, más de 500.000 bachilleres distribuidos en todo el territorio nacional (Ministerio del Poder Popular para la Educación Universitaria, 2004).

En tal sentido, la Misión Sucre se ofrecía para atender la urgencia nacional de la “...implementación de un sistema equitativo de ingreso a la educación superior, donde se hable de acceso en vez de selección, donde se universalice la oferta académica en los municipios, se creen nuevas modalidades de estudios y se flexibilicen los currícula [sic] y se reconozcan las competencias de las personas..." (Ministerio del Poder Popular para la Educación Universitaria, 2004: 24).

El objetivo prioritario de la Misión Sucre fue “...conformar y consolidar una red de todas aquellas instancias, instituciones y factores vinculados a la educación superior, que resuelva el problema del cupo universitario, a través de la generación de nuevos espacios y creación de nuevas modalidades de estudios convencionales y no convencionales." (Ministerio del Poder Popular para la Educación Universitaria, 2004: 25).

Para dar respuesta a la generación de nuevos espacios y, en el marco de la municipalización de la educación superior, la Misión Sucre asume la estrategia de las Aldeas Universitarias concebidas como “... centros municipales de Educación Superior donde confluyen los Programas de Formación (carreras) de distintas Instituciones, compartiendo recursos académicos (profesores, consultorías, espacios de reunión y discusión, laboratorios, centros de información y documentación, centros de práctica, actividades culturales, deportivas y de producción).” (Ministerio del Poder Popular para la Educación Universitaria, 2004: 50).

Otra de las políticas diseñadas durante esta etapa (2003-2006) fue la creación de la Universidad Bolivariana de Venezuela (UBV) en 2003, la cual se presentaba como un nuevo modelo de universidad, que desde el punto de vista conceptual, estructural y funcional debía ser diferente del resto de las universidades existentes en el país (Parra-Sandoval, Bozo de Carmona e Inciarte González, 2010). Su práctica tenía como principios orientadores: la responsabilidad con lo público, la ruptura con la concepción epistemológica de la estructura clásica de facultades, escuelas, cátedras, departamentos y el reconocimiento del diálogo como base del aprendizaje (Castellano, 2011). Para ello se propusieron un conjunto de estrategias que atenderían tanto el desempeño estudiantil con la creación del Programa de Iniciación Universitaria (PIUNI), como la selección e ingreso del personal docente a través del Programa de Inducción para el Ingreso Docente (PID) (Castellano, 2010).

Desde finales de 2004 hasta noviembre de 2006, un rasgo importante de esta etapa es el desmantelamiento de las políticas públicas en educación superior que habían sido diseñadas y parcialmente implementadas en el periodo anterior, tal como lo declara quien

\begin{tabular}{l|l|l} 
v.2 & n.1
\end{tabular}

p.67-88


fuera fundadora y primera rectora de la UBV, al señalar que “...las políticas y los proyectos que el Ministerio de Educación Superior venía desarrollando, no se continuaron, y en el caso de la Universidad Bolivariana, su proyecto fue desvirtuado." (Castellano, 2011: 353). Por su parte, otra de las personas que habían participado en el diseño e implementación de esas políticas se dirige al Presidente Chávez y con preocupación le expresa el peligro de "tirar a la basura los logros de la revolución" en materia de educación superior, y le advierte que los perjudicados por estas medidas serían los estudiantes becados para estudios de pregrado, los profesores becados para estudios de doctorado, los usuarios de las salas de computación y de las bibliotecas creadas por estos programas y los estudiantes indígenas de la Aldea Universitaria Bolivariana Gran Sabana (Scott, 2005).

Este desmantelamiento y la consecuente discontinuidad de las políticas puede haber influido en que el balance de esta etapa (2003-2006) revele que, aunque efectivamente puede atribuirse a la creación de la Misión Sucre y de la Universidad Bolivariana, así como al crecimiento exponencial de la Universidad Nacional Experimental Politécnica de la Fuerza Armada (UNEFA) ${ }^{13}$, el aumento de más del 100 por ciento de la matrícula estudiantil, que en el sector oficial pasó de 590.347 estudiantes de educación superior en 2003, a 1.218.434 en 2006, también es evidente que tal crecimiento no implicó una atención y cuidado paralelo a la calidad, en tanto los requisitos fundamentales para el desarrollo adecuado del proceso de formación de los estudiantes suponen: primero, la existencia de una infraestructura adecuada, la cual era inexistente en la medida en que fue necesario el uso de locales donde funcionan escuelas oficiales de educación básica, cuyas condiciones generalmente son bastante precarias; segundo, la participación de profesores de probada capacidad profesional y docente, imposible de adquirir con programas improvisados sobre la marcha, para atender el crecimiento de la población estudiantil; tercero, la implementación de estrategias de evaluación del aprendizaje que incorpore la autoevaluación, la co-evaluación y la heteroevaluación como se prevé (República Bolivariana de Venezuela, 2009), requiere no solo de la preparación y formación constante del docente, sino de la generación de un clima de responsabilidad y compromiso por parte de los estudiantes, que garantice confrontar los sesgos que estas modalidades de evaluación introducen si no son manejadas de manera adecuada, objetiva e imparcial, lo cual no parece factible ni a corto ni a mediano plazo, tanto por la improvisación del personal docente, último responsable del proceso de evaluación, como por la polarización política y la confrontación social existente en el país; y, finalmente, pero no menos importante, la generación de un clima de pluralidad y de respeto por las diferencias de cualquier índole y no de ideologización y politización, como lo que se propone con el eufemismo de la formación socio-política (Parra Sandoval, Bozo de Carmona e Inciarte González, 2010) para la formación de cuadros militantes del pensamiento único impuesto desde el gobierno (García Guadilla, 2011).

13 Esta institución es producto de la transformación del Instituto Universitario Politécnico de las Fuerzas Armadas Nacionales en Universidad.

\begin{tabular}{l|l|l|l|l|l|l} 
(C) Rev. Inter. Educ. Sup. & Campinas, SP & v.2 & n.1 & p.67-88 & jan./abr. 2016 & ISSN 2446-9424
\end{tabular}




\title{
Tercera etapa: 2007-2011
}

Es indudable que el año 2007 marcó de manera definitiva el proceso político que se había iniciado en 1999. Es a partir de entonces cuando, a pesar de no haber sido aprobada en referéndum la reforma constitucional propuesta por el Presidente Chávez y por la Asamblea Nacional, controlada totalmente por sus seguidores, para completar la transición a una "república socialista", esta se impone en el país. Así se decreta en el Proyecto Nacional Simón Bolívar. Primer Plan Socialista de Desarrollo Económico y Social de la Nación 20072013 (Gobierno Bolivariano de Venezuela, 2007), el cual pasa a constituirse en el documento base para la orientación de las políticas públicas elaboradas e implementadas a partir de ese momento.

En este sentido, en lo que tiene que ver con la educación superior, uno de los primeros indicadores del fortalecimiento de la orientación político-ideológica que el gobierno ha venido imprimiendo al proceso de refundación y reinstitucionalización de la República acentuado a partir de 2007- es el cambio de nombre del Ministerio de Educación Superior, el cual en 2006 pasa a llamarse Ministerio del Poder Popular para la Educación Superior (MPPEU); este cambio estuvo basado en

\begin{abstract}
...la clara necesidad de transformación de este órgano del Ejecutivo Nacional y de su ámbito de competencia, en función de la construcción y fortalecimiento del Poder Popular, como imperativo fundamental para la superación del modelo capitalista y la creación de una sociedad nueva, que ha de gestar su propio modelo: el Socialismo del Siglo XXI. ${ }^{14,}$ (Ministerio del Poder Popular para la Educación Universitaria, 2008a: 2).
\end{abstract}

Por otra parte, para reafirmar aún más el carácter socialista que el gobierno decide imprimir en todas sus instancias y acciones, el Ministerio de Educación Superior declara de manera taxativa que su Misión es ser:

...el órgano del Ejecutivo Nacional encargado de la dirección estratégica de la educación superior venezolana. Como tal, ejerce la rectoría del Sistema Nacional de Educación Superior y le corresponde la formulación, adopción, seguimiento y evaluación de las políticas y acciones dirigidas a garantizar una educación superior de calidad para todas y todos, que se constituya en factor estratégico para el fortalecimiento del poder popular y la construcción de una sociedad socialista ${ }^{15}$, democrática, participativa y protagónica. (Ministerio del Poder Popular para la Educación Universitaria, 2008a: 4).

Así mismo, declara que la Visión del Ministerio es:

Ser el órgano del Estado Socialista, al servicio del Poder Popular, que impulsa y regula, con visión estratégica, el desarrollo y transformación de la educación superior venezolana para garantizar el derecho de todos y todas a la participación en

\footnotetext{
${ }^{14}$ Subrayado nuestro.

${ }^{15}$ Subrayado nuestro. (C) Rev. Inter. Educ. Sup. Campinas, SP

\begin{tabular}{l|l|l} 
v.2 & n.1 & p.67-88
\end{tabular}
jan./abr. 2016 
la generación y socialización del conocimiento, en función del desarrollo endógeno, la soberanía nacional y la construcción de una sociedad socialista ${ }^{16}$, democrática, participativa y protagónica. (Ministerio del Poder Popular para la Educación Universitaria, 2008a: 4).

Nuevamente en 2009, se cambia el nombre a Ministerio del Poder Popular para la Educación Universitaria, a raíz de la aprobación de la Ley Orgánica de Educación, la cual introdujo esta denominación para los estudios de tercer nivel; en ese sentido, establece en el Artículo 25, el Sub-sistema de Educación Universitaria como parte del Sistema de Educación del país y en el Artículo 32 dispone las funciones y los principios rectores de la educación universitaria: su carácter público, la calidad y la innovación, el ejercicio del pensamiento crítico y reflexivo, la inclusión, la pertinencia, la formación integral, la formación a lo largo de toda la vida, la autonomía, la articulación y cooperación internacional, la democracia, la libertad, la solidaridad, la universalidad, la eficiencia, la justicia social, el respeto a los derechos humanos y la bioética así como la participación e igualdad de condiciones y oportunidades (Asamblea Nacional, 2009).

A partir de los mencionados principios, las políticas formuladas en el Plan Sectorial de Educación Superior 2008-2013 (Ministerio del Poder Popular para la Educación Superior, 2008b), basadas además en las Líneas Estratégicas del Plan Socialista, tienen los siguientes Objetivos Estratégicos:

$\checkmark \quad$ Universalizar la Educación Superior.

$\checkmark \quad$ Fortalecer las capacidades para la generación y socialización de conocimientos.

$\checkmark \quad$ Impulsar un nuevo modelo educativo, con centro en la formación ética, dirigido a la transformación social, la comprensión de nuestras realidades y entornos, la producción socialista y que de lugar a la diversidad de necesidades y expectativas de formación.

$\checkmark \quad$ Crear nuevas instituciones de educación superior y transformar las existentes.

$\checkmark \quad$ Municipalizar la Educación Superior, en estrecho vínculo con cada uno de los espacios y comunidades.

$\checkmark \quad$ Construir un sistema de educación superior, fundado en la cooperación solidaria, capaz de vincular los esfuerzos y recursos existentes.

$\checkmark \quad$ Fortalecer el papel de la educación superior en los procesos de integración latinoamericana y caribeña y como espacio para el desarrollo de vínculos solidarios con los pueblos del mundo.

Para el logro de estos objetivos estratégicos se propone un conjunto de políticas y acciones, entre las cuales cabe destacar:

${ }^{16}$ Subrayado nuestro.

(C) Rev. Inter. Educ. Sup.

Campinas, SP

v.2

n.1

p.67-88

jan./abr. 2016

ISSN 2446-9424 
a. Desarrollar un sistema nacional de ingreso a la educación superior con base a la equidad, que garantice el acceso de todos y todas a la educación superior;

b. Generar condiciones apropiadas para el buen desempeño estudiantil;

c. Construir, en conjunto con el Ministerio del Poder Popular para la Educación, un sistema nacional de orientación profesional;

d. Garantizar el derecho a una educación superior de calidad para las personas con discapacidad, los pueblos indígenas y las personas privadas de libertad;

e. Desarrollar los posgrados;

f. Apoyar la investigación;

g. Desarrollar un plan nacional de formación de los docentes de educación superior;

h. Desarrollar empresas socialistas universitarias;

i. Desarrollar un sistema nacional de evaluación y acreditación;

j. Construir criterios curriculares basados en la formación ética y la transformación social;

k. Fortalecer académicamente la Misión Sucre;

1. Crear comités locales y estadales de educación universitaria

m. Crear nuevas instituciones;

n. Crear el espacio de educación superior del ALBA.

En síntesis, se subraya como ha sido común en las etapas anteriores, la inclusión social y, en consecuencia, la universalización de la educación, pero además se plantea como uno de los objetivos a alcanzar durante el periodo (2007-2013), la adecuación de la educación superior al modelo productivo socialista.

En tal sentido, se oficializa en el año 2009 la creación de la Misión Alma Mater (Ministerio del Poder Popular para la Educación Universitaria, s. f.) la cual se constituye con la Misión Sucre -que había sido creada en 2003- en dos de las políticas dirigidas a lograr el mencionado objetivo general.

La Misión Alma Mater se propuso como metas la transformación de 29 Institutos y Colegios Universitarios Oficiales para dar origen a nuevas Universidades Nacionales Experimentales, la creación de 17 Universidades Territoriales, 10 Universidades Especializadas, 2 Institutos Especializados, la Universidad Bolivariana de Trabajadores "Jesús Rivero", la Universidad Nacional Experimental de los Pueblos del Sur y el fortalecimiento de la cobertura territorial de la educación universitaria a través de los Complejos Universitarios Socialistas Alma Mater (CUSAM).

De estas metas se lograron en esta etapa las siguientes: la Universidad Bolivariana de los Trabajadores "Jesús Rivero" (2008), la Universidad Nacional Experimental de las Artes (2008), la Universidad Nacional Experimental de la Seguridad (2009), el Instituto Latinoamericano de Agroecología (2008), 6 Universidades Politécnicas Territoriales (2010) y

\begin{tabular}{l|l|l|l|l|l|l} 
(C) Rev. Inter. Educ. Sup. & Campinas, SP & v.2 & n.1 & p.67-88 & jan./abr. 2016 & ISSN 2446-9424
\end{tabular}


el Instituto Universitario de Aeronáutica Civil (2009). Como institutos de estudios superiores se crearon el Centro Docente Cardiológico Bolivariano Aragua (2008) y la Escuela Venezolana de Planificación (2009).

De esta manera la plataforma institucional actual quedó conformada por:

$\begin{array}{ll}> & 58 \text { universidades } \\ & 17 \\ \checkmark & 5 \text { autónomas } \\ \checkmark & 28 \text { universidades experimentales oficiales } \\ \checkmark & 25 \text { universidades privadas } \\ > & 86 \text { institutos universitarios } \\ \checkmark & 27 \text { institutos universitarios oficiales } \\ \checkmark & 59 \text { institutos universitarios privados } \\ > & 16 \text { colegios universitarios } \\ \checkmark & 7 \text { colegios universitarios oficiales } \\ \checkmark & 9 \text { colegios universitarios privados } \\ > & 10 \text { institutos de estudios superiores } \\ \checkmark & 8 \text { institutos de estudios superiores oficiales } \\ \checkmark & 2 \text { institutos de estudios superiores privados }\end{array}$

Además coexisten con estas instituciones, las Aldeas Universitarias y los Complejos Universitarios Socialistas Alma Mater (CUSAM), que no aparecen registrados como instituciones de educación superior en la página del Ministerio, pero cuya existencia registra la Memoria y Cuenta de ese organismo ${ }^{18}$.

Este conjunto de instituciones da cuenta de un sistema de educación superior desarticulado, en tanto entre la mayoría de las instituciones oficiales, agrupadas en la Asociación de Rectores Bolivarianos (ARBOL) y directamente controladas por el gobierno y las agrupadas en la Asociación Venezolana de Rectores (AVERU) -universidades autónomas (5), experimentales dotadas de autonomía para elegir a sus autoridades (5) y las instituciones privadas (universidades, institutos y colegios universitarios)- no existen vasos comunicantes, ni puntos de encuentro, ni cualquier otra señal de consenso o al menos de comunicación, con relación a las políticas de educación superior y a la orientación de las transformaciones necesarias -cuyo reconocimiento es quizás el único punto compartido- haciendo inviable la posibilidad de constituir redes institucionales y mucho menos los comités locales y estadales de educación universitaria, con la participación igualitaria de todas las instituciones de educación superior de la localidad o del estado.

\footnotetext{
${ }^{17}$ Con relación a las universidades hay que destacar que por primera vez el número de universidades oficiales supera el de las privadas, no así en las demás instituciones -sobre todo institutos y colegios universitarios- en las cuales sigue predominando el sector privado.

${ }^{18} \mathrm{http} / / /$ www.mppeu.gob.ve/documentos/memoria_cuenta/2009_tomo1.pdf

\begin{tabular}{l|l|l|l|l|l|l} 
(C) Rev. Inter. Educ. Sup. & Campinas, SP & v.2 & n.1 & p.67-88 & jan./abr. 2016 & ISSN 2446-9424
\end{tabular}
}


Lo anterior confirma la crítica, tantas veces formulada, de la presencia de un sistema paralelo de educación universitaria, que responde más a la polarización política del país que a criterios estrictamente académicos, lo cual no contribuye a darle organicidad al conjunto de instituciones de educación superior, además de que tiende a inclinar la balanza hacia las instituciones agrupadas en ARBOL, no sólo en términos de la asignación de recursos sino en reconocimiento de la importancia de su papel, en desmedro de las universidades con mayor trayectoria y experiencia, presentes en AVERU. Esta segregación en el tratamiento de las instituciones de educación universitaria se evidencia en los órganos oficiales, a través de las páginas electrónicas, donde se da cuenta de los encuentros y reuniones de trabajo entre los representantes del MPPEU y los representantes (autoridades y sindicatos) de las instituciones identificadas con el gobierno nacional.

Sin embargo, hay que reconocer que el aumento en la cantidad de instituciones de educación universitaria puede ser una de las razones del incremento, más o menos constante, observado desde la etapa anterior en el número de estudiantes registrados, tal como se desprende de las cifras aportadas por el Ministerio del Poder Popular para la Educación Universitaria, las cuales dan cuenta de 2.015.140 estudiantes en 2007 y 2.120.231, en $2009^{19}$.

Por otra parte, este logro también responde a la implementación de la política de desarrollo del sistema nacional de ingreso a la educación superior. En el año 2008, se eliminó definitivamente la Prueba de Aptitud Académica (PAA), instrumento aplicado en el país desde 1984 como requisito obligatorio de ingreso a cualquier institución de educación superior, y se estableció un proceso de admisión llevado a cabo mediante dos mecanismos:

a. El Sistema Nacional de Ingreso a la Educación Universitaria (SINIEU) cuyo objetivo declarado es la "universalización e inclusión con pertinencia social" a través de la aplicación del Registro Único del Sistema Nacional de Ingreso a la Educación Universitaria (RUSNIEU), la Prueba Nacional de Exploración Vocacional (PNEV) con carácter obligatorio para los estudiantes del último año de educación secundaria y un modelo de asignación multivariable (http://www.mppeu.gob.ve/pni_info.php). Este modelo busca fundamentalmente aumentar la tasa de admisión de los estudiantes provenientes de los sectores menos favorecidos de la población, estratos IV y V, de acuerdo con la distribución del peso de las variables consideradas, tomando en cuenta el rendimiento académico en bachillerato, la regionalización para darle prioridad a los estudiantes provenientes de las zonas de influencia de la institución universitaria, y si el estudiante ha participado o no previamente en el proceso, con lo cual se busca subsanar la exclusión de quienes no hayan podido acceder antes al sistema

\footnotetext{
${ }^{19}$ La cifra provisional que ofrece el Ministerio del Poder Popular para la Educación Universitaria con relación a la matrícula para el año 2008 es de 2.109.331 y la estimada para 2009 es de 2.120.231; lo cual, comparado con la cobertura en 2002 que fue de 1.104.006 estudiantes, da cuenta de un crecimiento significativo de la matrícula de educación superior (Ministerio del Poder Popular para la Educación Universitaria, 2011).
}

\begin{tabular}{l|l} 
n.1 & p.67-88
\end{tabular}


(http://www.mppeu.gob.ve/documentos/boletin/SINIEEU.pdf). Mediante este proceso el MPPEU asume la responsabilidad de asignar en las universidades autónomas, al menos $30 \%$ de los cupos para nuevos ingresos y el $100 \%$ en las demás instituciones de educación universitaria oficiales.

b. Las cinco universidades autónomas y la Universidad Experimental Simón Bolívar para las cuales los requisitos del RUSNIEU y de la PNEV son de carácter obligatorio, exigen además la presentación de pruebas internas para la mayoría de las carreras y programas, con carácter obligatorio en la mayor parte de las instituciones. Las pruebas internas son de conocimiento y habilidades y, en el caso de la Universidad del Zulia (LUZ), esta es de carácter vocacional. Al mecanismo de las pruebas internas se añade el ingreso de estudiantes a la institución como respuesta a políticas institucionales de reconocimiento al mérito o excelencia académica, así como por el cumplimiento de compromisos asumidos con grupos internos de las propias instituciones como los profesores, empleados administrativos y obreros. Estas instituciones también cuentan con programas como el Samuel Robinson de la Universidad Central de Venezuela (UCV), el Fray Juan Ramos de Lora de la Universidad de Los Andes (ULA) y el de Admisión por Mérito Académico (AMA) de la Universidad del Zulia (LUZ), los cuales persiguen la incorporación de estudiantes provenientes de los sectores menos favorecidos de la población. Por otra parte, estas universidades también desarrollan programas conducentes a garantizar el acceso a estudiantes indígenas, a deportistas y a discapacitados. Mediante estos mecanismos las universidades autónomas se reservan el derecho de asignar el 70\% de los cupos en cada institución, después de recibir el 30\% asignado por el MPPEU.

Así, aunque el resultado de las políticas implementadas en esta tercera fase, de acuerdo con las cifras aportadas por el MPPEU, es el aumento de la cobertura, no sólo las cifras tendrían que ser corroboradas en las fuentes originales debido a la dificultad para obtenerlas y validarlas en el MPPEU ${ }^{20}$ y porque se manejan en términos del número de plazas ocupadas y no del número efectivo de estudiantes ${ }^{21}$, sino que hay que evaluar si, como en las etapas anteriores en las cuales se perseguía el mismo objetivo de universalización de la educación universitaria, para este acentuado proceso de crecimiento de la población estudiantil se ha previsto el cumplimiento de requisitos mínimos con relación a la calidad del aprendizaje, en términos del proceso de formación en sí mismo, de la calidad de los contenidos programáticos, de los recursos utilizados y de la infraestructura en general, y de la calidad del personal académico, para que la eficacia del proceso se exprese también en

\footnotetext{
${ }^{20}$ Hay que tener en cuenta que la validación de las cifras no puede ser por el avala que un organismo como la UNESCO pueda darles toda vez que instituciones de este tipo utiliza la información que aporta el propio gobierno.

${ }^{21}$ Se han encontrado casos en los cuales un mismo estudiante está inscrito en la UBV y al mismo tiempo cursa otra carrera en una universidad autónoma, o pertenece a la Misión Sucre (ya que allí recibe una beca) y estudia en otra universidad oficial. O cursa simultáneamente una carrera en una institución privada y en una oficial. En cada caso es registrado como un estudiante diferente.
}

\begin{tabular}{l|l|l|l|l|l|l} 
(C) Rev. Inter. Educ. Sup. & Campinas, SP & v.2 & n.1 & p.67-88 & jan./abr. 2016 & ISSN 2446-9424
\end{tabular}


equidad; así el incremento de la matricula sería efectivamente un logro en términos de la equidad.

En este sentido, una de las políticas propuestas para esta etapa fue la de desarrollar un sistema nacional de evaluación y acreditación, que podría ser la garantía de la inclusión con calidad.

Una de las estrategias implementadas, en términos del cumplimiento de esta política, fue la creación en 2008, de la Dirección General de Supervisión y Seguimiento de las Instituciones de Educación Superior (República Bolivariana de Venezuela. Presidencia de la República, 2008), la cual contempla entre sus funciones la aplicación de mecanismos de evaluación y seguimiento de la rendición de cuentas de las instituciones de educación superior; al mismo tiempo se creó el Comité de Evaluación y Acreditación de Programas e Instituciones de Educación Superior (CEAPIES), con la finalidad de coordinar los procesos de evaluación y acreditación institucional, para garantizar, reconocer y promover la calidad. De manera particular este Comité lleva adelante el proceso de acreditación de los programas seleccionados para participar en el marco del Sistema de Acreditación de Carreras Universitarias para el Reconocimiento Regional de la Calidad Académica de las Respectivas Titulaciones (ARCU-SUR).

Por otra parte, en 2011 se crea el Programa de Estímulo a la Investigación (PEI) cuyo objetivo es estimular y fomentar el desarrollo de la ciencia, tecnología e innovación, en las áreas prioritarias establecidas por el Estado (Observatorio Nacional de Ciencia, Tecnología e Innovación, 2011), lo cual se espera que redunde en la calidad de las instituciones universitarias. Este nuevo Programa pone el énfasis en la investigación colectiva y no en la producción individual del investigador, el cual debe enmarcar su trabajo en unas áreas definidas por el gobierno de acuerdo con el Primer Plan Socialista del Desarrollo Económico y Social para el periodo 2007-2013. Su evaluación se hará bajo criterios de equidad de género y de diversidad cultural, contribución a la soberanía nacional y al bienestar social, conceptos ambiguos que dependerán de la discrecionalidad del evaluador.

De tal manera que a pesar de los cambios introducidos y de los esfuerzos para imponer de manera permanente la evaluación como mecanismo de garantía de la calidad de la educación superior, no parece que se haya producido el efecto deseado en la dinámica de la educación universitaria venezolana.

Un marco legal hasta cierto punto difuso y volátil y altamente politizado, la inestabilidad en la continuidad de las políticas y la sustitución constante de funcionarios en los organismos encargados de la formulación e implementación de las políticas de educación superior, conforman un contexto en el cual es posible prever que a pesar de la creación de nuevas estructuras orientadas hacia la universalización de la educación superior, la transformación curricular y la evaluación, no hay en Venezuela, ni claridad en lo que se

\begin{tabular}{l|l|l|l|l|l|l}
\hline (C) Rev. Inter. Educ. Sup. & Campinas, SP & v.2 & n.1 & p.67-88 & jan./abr. 2016 & ISSN 2446-9424 \\
\hline
\end{tabular}


busca con relación a estos temas, ni el consenso necesario con relación a los mecanismos y procedimientos idóneos para lograr esos objetivos.

\section{A manera de conclusión: ¿hacia dónde van las políticas de educación superior en venezuela?}

En Venezuela, los hechos indican que la tendencia del núcleo conceptual de las políticas públicas implementadas en los últimos doce años, se orienta hacia más control e intervención del Estado y apuesta al establecimiento de condiciones que tienden a la paralización del mercado universitario; mientras que, en la mayor parte de la región e incluso del mundo, la tendencia que se observa está marcada por el discurso de la mercadización y su impacto en el rol del Estado, en su tratamiento de la educación superior. A esto hay que añadir que en Venezuela, la intervención del Estado se produce para transformar la educación universitaria en función de la imposición de una corriente ideológica: el socialismo. Ello supone la sustitución de una supuesta forma de pensamiento único por otra, y niega la pluralidad y el respeto por la diferencia que, teóricamente forma parte de los principios que sustentan las políticas públicas de educación superior en el periodo 1999-2011.

Por otra parte, el desarrollo de estas políticas bajo el principio de la universalización de la educación universitaria ha obviado que no puede hablarse de equidad, si ésta no está acompañada de exigencias de calidad, que garanticen que, efectivamente sea posible superar las diferencias basadas en condición socio-económica de las personas.

Finalmente, todo parece indicar que el clima de polarización política, que conduce a la segregación, continuará presente en la orientación de las políticas públicas en educación superior. Siendo el año 2012 un año electoral, es posible prever que las tensiones entre los dos grandes sectores de la sociedad venezolana: el gobierno y sus seguidores y la oposición se agudizarán; sin embargo, también es posible prever que, en función de intereses electorales, el gobierno no promueva políticas que puedan producir situaciones límite en las tensiones que tal confrontación pueda llevar a la educación superior. En otras palabras, las transformaciones que se promuevan en el sector para responder al proyecto socialista, se caracterizarán por su introducción paulatina, tratando de no generar situaciones extremas que puedan distorsionar las políticas, o generar disturbios en el país dado el momento electoral. 


\section{Referencias}

ASAMBLEA NACIONAL.. Ley Orgánica de Educación. Gaceta Oficial de la República Bolivariana de Venezuela. n. 5.929. 2009

CASTELLANO, María Egilda. Introducción. In: MINISTERIO DE EDUCACIÓN, CULTURA Y DEPORTES. Políticas y estrategias para el desarrollo de la educación superior en Venezuela 2000-2006. 2001. Disponivel en: 〈http://www.mes.gob.ve/mes/documentos/ descarga/politicas_estrategias.pdf $>$. Acesso en: 20 jun. 2010.

CASTELLANO, María Egilda. Experiencias de transformación universitaria en Venezuela. In: CADENAS, José María (Coord.). La universidad latinoamericana en discusión. Caracas: UCV-UNESCO-IESALC, 2010.

CASTELLANO, María Egilda. Educación Universitaria en Venezuela, 2000-2010: logros y Compromisos. Espacio Abierto, v.20, n.2, p.343-365, abr./jun. 2011.

Consejo de Redacción. (2004, Diciembre). Entrevista a María Egilda Castellano fundadora y ex-rectora de la Universidad Bolivariana de Venezuela. Revista Laberinto, 16, Artículo 6. Consultada 23 de Octubre, 2009 en http://laberinto.uma.es/index.php?option=com_content\&view=article\&id=214:entrevista-amaria-egilda-castellano-fundadora-y-ex-rectora-de-la-universidad-bolivariana-devenezuela\&catid $=50: 1 a b 16 \&$ Itemid $=54$

Cox, Cristián (1993) Políticas de Educación Superior: Categorías para su análisis. En Courard, Hernán (Editor) Políticas Comparadas de Educación Superior en América Latina. Santiago, Chile: FLACSO.

De Venanzi, Augusto (2010). Authoritarianism versus welfare policy: the two faces of the bolivarian revolution. Revista Venezolana de Análisis de Coyuntura, XVI, 53-76.

García Guadilla, Carmen (2005) Políticas públicas de educación superior en Venezuela (1999-2004). En Centro de Estudios del Desarrollo (CENDES) Venezuela Visión Plural. Una mirada desde el CENDES. Caracas: bid \& co. Editor c.a. Pp. 184-208.

García Guadilla, Carmen (2006) Proyecto CINDA. Educación Superior en Iberoamérica 2006. Informe de Venezuela.

García Guadilla, Carmen (2011) Tensiones de la educación superior venezolana en un contexto de explicita polarización política. En Tensiones de la educación superior venezolana en un contexto de explicita polarización política \& Resultados de un debate. Temas para la discusión. Caracas: CENDES.

Gobierno Bolivariano de Venezuela. (2007). Proyecto Nacional Simón Bolívar. Primer Plan socialista. Desarrollo Económico y Social de la Nación 2007-2013. Caracas: Presidencia. 
Fuenmayor Toro, Luis (2004) La Aldea Universitaria Bolivariana. Página Digital. Consultada el 30 de junio de 2011 en

http://www.paginadigital.com.ar/articulos/2004/2004cuart/educacion/e1422610-4.asp

González Silva, Humberto (2008). La Educación Superior en la Revolución Bolivariana. Documento mimeografiado. Caracas.

López Maya, Margarita. Apuntes sobre la polarización política en Venezuela y los países andinos (Tema Central) En: Ecuador Debate. ¿La democracia en sus límites?, Quito: Centro Andino de Acción Popular CAAP, (no. 80, agosto 2010): pp. 95-104.

Ministerio de Educación, Cultura y Deportes (2001) Políticas y Estrategias para el desarrollo de la Educación Superior en Venezuela 2000-2006. Consultada 20 de junio de 2010. http://www.mes.gob.ve/mes/documentos/descarga/politicas_estrategias.pdf

Ministerio del Poder Popular para la Educación Superior (2008). Plan Sectorial para la Educación Superior 2008-2013. Consultada 13 de abril de 2009

http://www.mppeu.gob.ve/documentos/descarga/plan_sectorial_191108.pdf.

Ministerio del Poder Popular para la Educación Universitaria (2004) Misión Sucre Compendio Documental Básico. Consultada 13 de abril de 2009 http://www.mppeu.gob.ve/documentos/descarga/pdf21-12-2009_08:54:06.pdf

Ministerio del Poder Popular para la Educación Universitaria (2008a). Sistema Nacional de Ingreso a la Educación Universitaria en Venezuela. Documento Oficial. DOP-2008-02.

Ministerio del Poder Popular para la Educación Superior (2008b) Plan Sectorial 2008-2013.

Ministerio del Poder Popular para la Educación Universitaria (s. f.). Misión Alma Mater. Educación Universitaria Bolivariana y Socialista. http://www.mes.gov.ve/documentos/descarga/pdf21-12-2009_07:30:40.pdf

Ministerio del Poder Popular para la Educación Universitaria (2011). Logros de la Revolución Socialista Bolivariana en Educación Universitaria 1999-2010. Consultada 20 de julio de 2011

http://www.mppeu.gob.ve/web/uploads/PDF/FOLLETO_LOGROS_DE_LA_REVOLUCIO N_SOCIALISTA_BOLIVARIANA_EN_EDUCACION_UNIVERSITARIA.pdf

Mundó, Mabel (2009). Las misiones educativas: ¿política pública para la inclusión o estrategia para el clientelismo político? Cuadernos del CENDES, 26, 27-65.

Navarro Díaz, Héctor (2001) Presentación. En Ministerio de Educación, Cultura y Deportes (2001) Políticas y Estrategias para el desarrollo de la Educación Superior en Venezuela 20002006. Consultada 20 de junio de 2010

http://www.mes.gob.ve/mes/documentos/descarga/politicas_estrategias.pdf

Observatorio Nacional de Ciencia, Tecnología e Innovación (2011) Reglamento del Programa de Estímulo a la Investigación. http://www.oncti.gob.ve/oncti/

\begin{tabular}{l|l} 
n.1 & p.67-88
\end{tabular}


Parra-Sandoval, María Cristina (2010a). Las transformaciones de la educación superior en Venezuela: en búsqueda de su identidad. Revista Educación Superior y Sociedad. Vol. 15, $\mathrm{N}^{\circ}$ $1,107-128$.

Parra-Sandoval, Maria Cristina (2010b). El sistema de evaluación del posgrado y de la evaluación. En Ferreira de Oliveira, J. Mendes Catani, A. y Carapeto Ferreira, N. S. Pósgraduação e Avaliação. Impactos e perspectivas no Brasil e no cenário internacional. Brasil: Mercado de Letras, 257-284.

Parra-Sandoval, María Cristina, Bozo de Carmona, Ana Julia e Inciarte González, Alicia (2010) University: The Last Call? Maracaibo: Ediciones Astro Data.

República Bolivariana de Venezuela (1999). Constitución Nacional de la República Bolivariana de Venezuela.

República Bolivariana de Venezuela. Ministerio de Educación Superior. Consejo Nacional de Universidades. Oficina de Planificación del Sector Universitario (2002). Proyecto “Alma Mater" para el Mejoramiento de la Calidad y la Equidad de la Educación Universitaria en Venezuela. Desempeño Estudiantil y Equidad en la Universidad Venezolana. Apoyo Académico al Desempeño Estudiantil (PRODES). Caracas: Editorial Cuadernos OPSU.

República Bolivariana de Venezuela. Presidencia de la República (2002). Decreto N 1.634. Reforma del Decreto sobre Organización y Funcionamiento de la Administración Pública Central. Gaceta Oficial de la República Bolivariana de Venezuela $\mathrm{N}^{\circ}$ 37.359. Consultada el 01 de Julio de 2009 en http://www.tsj.gov.ve/gaceta/enero/080102/080102-37359-16.html

República Bolivariana de Venezuela. Presidencia de la República (2008) Reglamento Orgánico del Ministerio del Poder Popular para la Educación Superior. Gaceta Oficial de la República Bolivariana de Venezuela $\mathrm{N}^{\circ}$ 39.032. Consultada 7 de marzo de 2011. http://www.mppeu.gob.ve/web/uploads/PDF/reglamento_mppes-1.pdf

República Bolivariana de Venezuela. Ministerio del Poder Popular para la Educación Superior. Universidad Bolivariana de Venezuela. Consejo Universitario (2009). Reglamento General de Valoración del Desempeño Estudiantil de la Universidad Bolivariana de Venezuela. Consultada 2 de marzo de 2010 http://www.scribd.com/doc/45554416/REGLAMENTO-DEl-DESEMPENO-ESTUDIANTIL

Sott, Tania (2005) Carta abierta al compatriota Hugo Chávez Frías. Aporrea.org. Consultada 10 de junio de 2011. http://www.aporrea.org/educacion/a12571.html 